\title{
The interaction between 5-HTTLPR and stress exposure influences connectivity of the executive control and default mode brain networks
}

\author{
Dennis van der Meer ${ }^{1}$ - Catharina A. Hartman ${ }^{1}$. Raimon H. R. Pruim ${ }^{2,3}$. \\ Maarten Mennes ${ }^{2,3}$ • Dirk Heslenfeld ${ }^{4}$. Jaap Oosterlaan ${ }^{4}$ - Stephen V. Faraone ${ }^{5,6}$. \\ Barbara Franke $^{7}$ • Jan K. Buitelaar ${ }^{2,3,8}$ • Pieter J. Hoekstra ${ }^{1}$
}

Published online: 13 October 2016

(C) The Author(s) 2016. This article is published with open access at Springerlink.com

\begin{abstract}
We recently reported that the serotonin transporter polymorphism 5-HTTLPR moderates the relation between stress exposure and attention-deficit/hyperactivity disorder (ADHD) severity. This gene-environment interaction (GxE) has been previously tied to the processing of emotional stimuli, which is increasingly recognized to be a key factor in ADHD-related impairment. The executive control and default mode brain networks play an important role in the regulation of emotion processing, and altered connectivity of these networks has also been associated with ADHD. We therefore investigated whether resting-state connectivity of either of these networks mediates the relation of 5-HTTLPR and stress exposure with ADHD severity. Resting-state functional magnetic resonance imaging, genetic, and stress exposure questionnaire data was available for 425 adolescents and young adults (average age 17.2 years). We found that 5-HTTLPR Sallele carriers showed a more negative relation between stress exposure and connectivity of the executive control network
\end{abstract}

Jan K. Buitelaar and Pieter J. Hoekstra shared last author.

Electronic supplementary material The online version of this article (doi:10.1007/s11682-016-9633-3) contains supplementary material, which is available to authorized users.

Dennis van der Meer dvdm09@gmail.com

1 Department of Psychiatry, University Medical Center Groningen, University of Groningen, P.O. Box 30001, 9700 RB, Groningen, The Netherlands

2 Department of Cognitive Neuroscience, Donders Institute for Brain, Cognition and Behaviour, Radboudumc, Nijmegen, The Netherlands

3 Centre for Cognitive Neuroimaging, Donders Institute for Brain, Cognition and Behaviour, Radboudumc, Nijmegen, The Netherlands than L-allele homozygotes, specifically in the pre/postcentral gyrus, striatum, and frontal pole. In the default mode network, we found a positive association between the GxE and supramarginal gyrus connectivity. Connectivity of either network did not significantly mediate the effect of this GxE on ADHD. Opposite effects of stress exposure on connectivity in the executive and default mode networks may contribute to findings that stress exposure is associated with lowered cognitive control and heightened levels of rumination and worrying, for S-allele carriers but not L-allele homozygotes. When combined, these effects on connectivity of both networks may relate to the emotional problems seen in individuals with ADHD.

Keywords Serotonin transporter · Psychological stress · Attention-deficit/hyperactivity disorder · Gene-environment interaction · Functional magnetic resonance imaging

4 Department of Clinical Neuropsychology, VU University Amsterdam, Amsterdam, the Netherlands

5 Departments of Psychiatry and of Neuroscience and Physiology, SUNY Upstate Medical University, Syracuse, USA

6 K.G. Jebsen Centre for Psychiatric Disorders, Department of Biomedicine, University of Bergen, Bergen, Norway

7 Departments of Human Genetics and Psychiatry, Donders Institute for Brain, Cognition and Behaviour, Radboudumc, Nijmegen, The Netherlands

8 Karakter Child and Adolescent Psychiatry University Centre, Nijmegen, The Netherlands 


\section{Introduction}

Our genetic make-up influences how we respond to environmental factors. The study of genes moderating the effect of environmental risk factors for psychiatric disorders may therefore lead to a better understanding of the etiology of these disorders than studying genetic and environmental factors in isolation (Caspi and Moffitt 2006). The most investigated gene-environment interaction $(\mathrm{GxE})$ in psychiatry is the interaction between a polymorphism in the promoter region of the serotonin transporter gene $(5-H T T L P R)$ and exposure to psychosocial stress. Numerous papers have reported that people carrying the short variant (S-allele) of 5-HTTLPR have a stronger association between stress exposure and psychiatric disorders than people homozygous for the long variant of this gene (L-allele; Caspi et al. 2010), and animal models have provided evidence of a causal relation of this GxE with a range of pathological behaviors (Spinelli et al. 2007).

We recently found that S-allele carriers have a stronger relation between stress and ADHD severity than L-allele homozygotes, independent of internalizing comorbidity (van der Meer et al. 2014). On the other hand, a meta-analysis has found that on average L-allele homozygotes have a higher risk of ADHD (Gizer et al. 2009). S-allele carriers and L-allele homozygotes differ in the detection and subsequent processing of stimuli, particularly emotional ones, which may explain the role of 5-HTTLPR, and its interaction with stress exposure, in ADHD. Several studies have shown that S-allele carriers outperform L-allele homozygotes on a range of neuropsychological tasks (Borg et al. 2009; Roiser et al. 2007), while others have rather reported lower performance (Owens et al. 2012; Fischer et al. 2015). Higher sensitivity to stimuli by Sallele carriers may explain the heterogeneity of findings, as this would be beneficial under positive conditions while having a negative effect under stressful, emotional conditions (Homberg and Lesch 2011). S-allele carriers show higher levels of neuroticism (Lesch et al. 1996), rumination (Clasen et al. 2011), and cognitive reactivity (Wells et al. 2010), a tendency for dysfunctional thinking when exposed to stressors (Barnhofer and Chittka 2010). Combined with an oftenreported attention bias to negatively-valenced information (Pergamin-Hight et al. 2012), these differences in the processing of stimuli are likely to contribute to the stronger relation between stress exposure and psychopathology for S-allele carriers than for L-allele homozygotes. There is considerable overlap between the behavioral correlates of the S-allele and ADHD; results from a range of studies suggest that individuals with ADHD are also more sensitive to stimuli. They have been shown to respond stronger to positive reinforcement than healthy individuals (Luman et al. 2005), but also to have a tendency to orient less towards positive than negative emotional stimuli (Shaw et al. 2014), to be distracted more by negative emotional information in cognitive tasks (Posner et al. 2011), to score higher on measures of neuroticism (Martel et al. 2009; Parker et al. 2004), and to have more negative automatic thoughts (Mitchell et al. 2013).

Neuroimaging studies of 5-HTTLPR, and its interaction with stress, have provided clues which neural pathways may mediate the effects of this GxE on behavior. The majority of studies into 5-HTTLPR have employed a region-of-interest approach, focusing on the activity of the amygdala and associated limbic regions when exposed to an acute stressor. These studies have mostly found higher activity of these regions in S-allele carriers (Munafo et al. 2008). Pezawas et al. reported that the association between 5-HTTLPR and anxiety is partly explained by lowered top-down control of the anterior cingulate cortex over the amygdala when faced with stressful stimuli (Pezawas et al. 2005). Whole-brain investigations have reported that S-allele carriers show stronger effects of longterm stress exposure on the structure and activity of frontal brain regions, particularly the anterior cingulate cortex, than L-allele homozygotes (Canli et al. 2006; Selvaraj et al. 2011). In line with this literature, we recently reported that gray matter volume in the anterior cingulate cortex and frontal pole mediates the association of the interaction between 5-HTTLPR and stress exposure with ADHD severity (van der Meer et al. 2015). Together, these findings suggest that the heightened sensitivity to stress by Sallele carriers may involve both enhanced emotional reactivity by limbic structures and lower cognitive control by frontal cortical structures.

Our understanding of the neural mechanisms underlying 5-HTTLPR, and its interaction with stress exposure, may be increased by studying measures of functional network connectivity, as proper serotonin signaling has been shown to be crucial for the development of fundamental neural networks (Sodhi and Sanders-Bush 2004). Functional connectivity networks are sets of brain regions that have highly correlated activity patterns, thought to reflect a shared function. Through independent component analysis (ICA), Smith et al. have identified a set of intrinsic connectivity networks during resting conditions which could be unambiguously matched to brain networks found when averaging over activity patterns from a large amount of functional magnetic resonance imaging (fMRI) studies during a range of tasks (Smith et al. 2009), thereby providing a wealth of information on their behavioral correlates. These networks can be identified with high reliability and replicability (Damoiseaux et al. 2006). Further, network connectivity measures have been postulated to better account for behavioral effects of genetic variation than local measures of brain activity or structure (MeyerLindenberg 2009), and have proven to be a powerful tool in the study of psychiatric disorders, including ADHD (Oldehinkel et al. 2013; Broyd et al. 2009). 
Given that differences between S-allele carriers and Lallele homozygotes in the processing of emotional stimuli may explain the interaction between 5-HTTLPR and stress exposure on ADHD, two of the networks identified by Smith et al. are of particular interest for studying this GxE: the executive control network and the default mode network. The executive control network covers several medial-frontal areas and the basal ganglia, regions richly innervated by serotonergic neurons (Puig and Gulledge 2011) and often linked to ADHD in both task-based and resting-state fMRI (rs-fMRI) analyses (Oldehinkel et al. 2013; Castellanos et al. 2008; Bush et al. 1999). The two brain regions we previously reported to mediate the association of 5-HTTLPR and stress with ADHD, the frontal pole and anterior cingulate cortex (van der Meer et al. 2015), are also part of this network. It is most strongly activated during cognitive tasks, and is associated with the behavioral domains 'action-inhibition', 'emotion', and 'perception-somesthesis-pain' (Smith et al. 2009). The default mode network consists of inferior lateral and medial parietal regions, as well as the ventromedial frontal cortex. Its activity has been shown to be dependent on serotonin availability (Kunisato et al. 2011). It is the most extensively studied network due to its apparent deactivation during cognitive tasks, sparking discussion on the existence of a default mode of brain functioning (Raichle et al. 2001). This network is associated with self-referential cognitive processes, e.g., using past experiences to plan future actions (Buckner et al. 2008). Heightened default mode network activity and connectivity is associated with negative rumination (Whitfield-Gabrieli and Ford 2012). Lowered suppression of this network during external attention-demanding tasks has been suggested to contribute to problems with cognitive performance of individuals with ADHD, through interfering, task-irrelevant, thoughts leading to lapses of attention (Sonuga-Barke and Castellanos 2007).

In the present study, we aimed to determine whether 5HTTLPR genotype moderates the association of stress exposure with functional connectivity in the executive control and default mode networks, and whether connectivity in either of these networks mediate the effect of this GxE on ADHD severity. We therefore performed two mediation analyses, with the GxE as predictor, functional connectivity maps of either the executive control or the default brain network as mediator, and ADHD symptom count as outcome. The analyses were carried out in a sample of adolescents and young adults (mean age 17.2 years) consisting of individuals with and without ADHD, thus enabling analysis within a wide range of ADHD severity, in accordance with the continuous distribution of ADHD within the population (Levy et al. 1997). In this cohort we have previously shown that the interaction between 5-HTTLPR genotype and stress exposure predicts ADHD severity (van der Meer et al. 2014).

\section{Materials and methods}

Participants were selected from the NeuroIMAGE study, a follow-up of the Dutch part of the International Multicenter ADHD Genetics (IMAGE) study (von Rhein et al. 2014). NeuroIMAGE included 365 families with at least one child with ADHD and at least one biological sibling (regardless of ADHD diagnosis) and 148 control families with at least one child, without any formal or suspected ADHD diagnosis in any of the first-degree family members. ADHD families were recruited through ADHD outpatient clinics in the regions Amsterdam, Groningen, and Nijmegen (the Netherlands). Control families were recruited through primary and high schools in the same geographical regions. To be included in NeuroIMAGE, participants had to be of European Caucasian descent, between ages 5 and 30 , have an IQ $\geq 70$, and no diagnosis of autism, epilepsy, general learning difficulties, brain disorders, or known genetic disorders. More information on the NeuroIMAGE study and its participants is available elsewhere (von Rhein et al. 2014).

\section{Measurements}

All measurements were part of a comprehensive assessment protocol. Testing was carried out either at the VU University Amsterdam and VU University Medical Centre or at the Radboud University Nijmegen Medical Centre and Donders Institute for Brain, Cognition, and Behavior in Nijmegen. Participants were asked to withhold use of psychoactive drugs for $48 \mathrm{~h}$ before measurement. During the testing day, participants were motivated with short breaks and received $€ 50$ and a copy of their MRI scan at the end of the day. The study was approved by the regional ethics committee (CMO Regio Arnhem - Nijmegen; 2008/163; ABR: NL23894.091.08) and the medical ethical committee of the VU University Medical Center. All participants signed informed consent (for participants between 12 and 18 years of age, both the parents and participant signed, for participants under 12 only the parents signed).

After selection of those who met the inclusion criteria and had complete phenotypic and good quality rs-fMRI data available, our sample consisted of 425 participants from 255 families. This sample contained 161 participants with a full diagnosis of ADHD, 53 participants with subthreshold ADHD (i.e., ADHD symptoms without meeting the criteria for a full ADHD diagnosis, see the Online resources for a definition), and 207 participants with no ADHD diagnosis. Diagnoses were made in accordance with Diagnostic and Statistical Manual (DSM) IVTR criteria on the basis of a combination of a semi-structured diagnostic interview, the Kiddie Schedule for Affective Disorders and Schizophrenia - Present and Lifetime version (Kaufman et al. 1997), and the Conners Rating Scales. In this sample, 53 participants had an oppositional defiant disorder or 
conduct disorder, 12 an internalizing disorder, and 66 reading disorder. An extensive description of the diagnostic algorithm for ADHD and comorbid disorders is provided in the Online resources.

As a measure of ADHD severity, we constructed an ADHD symptom count based on the Conners ADHD Rating Scales questionnaires (Conners et al. 1998). These questionnaires were filled in by the parents and either a teacher (for participants $<18$ years) or the participants themselves (for those $\geq 18$ years old). The Conners Rating Scales provide operational definitions of each of the 18 ADHD symptoms defined by the DSM-IV-TR. In this sample, the symptom count ranged from 0 to 18 with an average of 5.1 (standard deviation (SD) 5.0). For more details on how this measure was constructed, see the Online resources.

Two questionnaires were used to assess the amount of exposure to psychosocial stress, as described in previous work (van der Meer et al. 2014). Parents filled in the Long-Term Difficulties (LTD) questionnaire (Bosch et al. 2012; Oldehinkel et al. 2008), which contained thirteen items measuring whether their children have been exposed to chronic stressors such as a handicap, being bullied, having financial difficulties, or other persisting problems at home or school. They were asked to only report chronic, ongoing difficulties. In addition, participants themselves filled in a Stressful Live Events (SLE) questionnaire (Bosch et al. 2012; Oldehinkel et al. 2008), which contained eleven items on exposure to specific major stressful events in the past 5 years, such as death or serious illness of a loved one, physical or sexual abuse, or failure at something important to them. For the composite stress measure, the scores on these questionnaires were transformed to Z-values and averaged according to common practice for aggregating similar measures, as previously described elsewhere (van der Meer et al. 2014). See the Online resources for further information on both questionnaires and an overview of the items.

Genotyping was performed as described in Brookes et al. (2006) and in the Online resources. Briefly, DNA was extracted from blood samples at Rutgers University Cell and DNA Repository, New Jersey, USA. Standard polymerase chain reaction protocols were used for the determination of 5 $H T T L P R$ genotype. This study investigated a dominant genetic model of the 5-HTTLPR S-allele, wherein S-allele carriers were coded as ' 1 ' and L-allele homozygotes were coded as ' 0 '. This is in accordance with the majority of studies investigating this GxE (Caspi et al. 2010) and is based on the functional effects of the S- and L-alleles (Lesch et al. 1996). In addition, L-alleles with the rs $25531 \mathrm{C}-\mathrm{G}$ single nucleotide polymorphism were recoded as a functional S-allele, in accordance with prior studies (Hu et al. 2006). This led to $20 \mathrm{~L}$ allele homozygotes being recoded to S-allele carriers. Genotype frequencies did not deviate from Hardy-Weinberg Equilibrium $\left(\chi^{2}=0.66, p=.42\right)$.
As a measure of socio-economic status, the highest successfully completed education level of the parents was recoded into a measure reflecting years of education. This scale contained nine levels, ranging from 0 (no formal education) to 17 (university) years of education (Buis 2010). The average of both parents was used, which, in this sample, ranged from 5 to 17 with an average of 12.1 (SD 2.5).

\section{Resting-state fMRI data processing}

All subjects were scanned with either a Siemens MAGNETOM Sonata 1.5 Tesla (at VU UMC in Amsterdam) or a Siemens MAGNETOM Avanto 1.5 Tesla (at Donders Centre for Cognitive Neuroimaging in Nijmegen) MRI scanner (Siemens, Erlangen, Germany), using identical protocols. Functional images during rest were obtained using a gradient echo echo-planar imaging (GE-EPI) sequence $(\mathrm{TR}=1960 \mathrm{~ms}$, $\mathrm{TE}=40 \mathrm{~ms}, \mathrm{FOV}=224 \mathrm{~mm}, 37$ axial slices, flip angle $=80$, matrix size $=64 \times 64$, in-plane resolution $=3.5 \mathrm{~mm}$, slice thickness $/$ gap $=3.0 \mathrm{~mm} / 0.5 \mathrm{~mm}, 266$ volumes). Participants were instructed to relax with their eyes open during the rsfMRI scan, which lasted 8 minutes.

Preprocessing consisted of removal of the first five volumes, primary head movement correction by realignment to the middle volume, global 4D mean intensity normalization and spatial smoothing with a $6 \mathrm{~mm}$ Gaussian kernel. We corrected for secondary effects of head motion by applying ICA-AROMA, a robust ICA-based strategy for automatic detection and removal of motion-related artifacts (Pruim et al. 2015). We additionally removed signal from white matter and cerebrospinal fluid using nuisance regression, and applied temporal high-pass filtering $(>0.01 \mathrm{~Hz})$. We co-registered the functional data to the participant's structural image using affine boundary-based registration as implemented in FSL FLIRT (Jenkinson et al. 2002) and subsequently transformed them to MNI152 standard space with $4 \mathrm{~mm}$ isotropic resolution using non-linear registration through FSL FNIRT.

Starting out with 486 participants who had behavioral and rs-fMRI data available, we first excluded the top $5 \%$ of participants with highest motion score $(n=24)$, as calculated by the root mean squared of the frame-wise displacement time series. Subsequently, 19 individuals were removed based on scan quality (e.g. artifacts, less than 240 volumes available, insufficient coverage of the entire brain), and 18 due to incidental findings after visual inspection (e.g. enlarged ventricles or unexpected hypo-intensities), for our final sample size of $n=425$.

For each participant, we derived spatial maps of the executive control and default mode networks using dual regression (Beckmann et al. 2009). This consisted of a multivariate spatial regression of a set of initial templates against the preprocessed rs-fMRI data, yielding participant-specific time series. The resulting time series were then entered in a 
multivariate temporal regression against the same preprocessed rs-fMRI data resulting in participant-level spatial representations of the initial templates (Beckmann et al. 2009; Filippini et al. 2009).

As initial template set, we used the twenty-dimensional resting-state components described by Smith et al. (2009), which have been made publicly available. We chose these because they have been shown to closely correspond to brain networks identified in thousands of individuals across a wide range of tasks (Smith et al. 2009). Use of these templates allows for better comparison between studies and comes with a large amount of information on the networks' behavioral correlates from previous studies (Laird et al. 2011; Smith et al. 2009).

\section{Mediation analyses}

For the mediation analyses, we employed Mediation Effect Parametric Mapping (Wager et al. 2008). This analysis technique is based on a standard three-variable mediation model, investigating the association of the predictor $\mathrm{X}$ with the mediator $\mathrm{M}$, and the association of mediator $\mathrm{M}$ with the dependent variable $\mathrm{Y}$. The mediation effect, i.e., the effect of $\mathrm{X}$ on $\mathrm{Y}$ mediated by $\mathrm{M}$, is the product of the resulting two regression coefficients, the significance of which is determined through bootstrapping (Hayes 2013). We ran two mediation models, one for either network. Both consisted of 5-HTTLPR genotype, amount of stress exposure, and their interaction as predictors, the map resulting from the output of the dual regression on the executive control or default mode network template as mediator, and ADHD symptom count as dependent variable. We included sex, age, scanner location, and socio-economic status as covariates. All predictors and covariates were mean-centered. The mediation analyses were performed in MATLAB with the Multilevel Mediation and Moderation Toolbox (Wager et al. 2008), which performed bootstrapping (5000 samples) on each voxel. Family-wise error correction was applied through the use of FSL's EasyThresh, which carries out cluster-based thresholding. A Z-value of 2.3 was used to define contiguous clusters and subsequently, each cluster's significance level was estimated on the basis of Gaussian Random Field theory. Those clusters surviving a significance threshold of $p=.01$ are reported. We used the Harvard-Oxford atlas for localization. All reported coordinates are in MNIspace and in millimeter.

For plotting of the results and calculation of summary statistics reported in Table 2, we used R, v3.1.1. (R CoreTeam 2012). For every participant we extracted the mean network connectivity from the clusters identified in the main analyses. We reran the analyses with linear mixed effects models, estimating a random intercept for family. In addition to 5-HTTLPR, stress exposure, and their interaction, all models had sex, age, scanner location, and socio-economic status included as covariates. We calculated Cohen's $f^{2}$ as a measure of effect size for the significant predictors (Selya et al. 2012), see Table 2.

\section{Sensitivity analyses}

We checked whether the direction of effects was the same across diagnostic status, testing locations, and age groups, to ensure findings were not driven by any one group. More information on the methods for these analyses can be found in the Online resources.

\section{Results}

No significant differences in stress exposure, sex, age, testing location, socio-economic status or head motion during scanning were found between S-allele carriers and L-allele homozygotes, as summarized in Table 1.

5-HTTLPR genotype significantly moderated the effect of stress exposure on ADHD symptom count $(\mathrm{B}=1.65$, $\mathrm{SE}=0.61, p=.007)$, as previously reported in a sample from which the current sample is a subset (van der Meer et al. 2014). The conditional effects of genotype or stress exposure on ADHD symptom count were not significant. Within-group analysis confirmed that stress exposure was highly significantly related to ADHD symptom count in S-allele carriers $(\mathrm{B}=2.07, \mathrm{SE}=0.37, p<.0001)$, but not in L-allele homozygotes $(\mathrm{B}=0.58, \mathrm{SE}=0.53, p=.28)$.

\section{Executive control network connectivity}

The executive control network covers large portions of the medial frontal lobe, frontal pole, and basal ganglia. The corresponding brain map is displayed in the Online resources.

The association between stress and connectivity of regions in the executive control network was moderated by 5 HTTLPR genotype bilaterally in the precentral gyrus extending into the postcentral gyrus, right frontal pole, left thalamus and caudate nucleus (see Fig. 1). S-allele carriers had a more negative correlation between stress and connectivity than Lallele homozygotes in all four clusters (see Fig. 2A and Table 2).

\section{Default mode network connectivity}

The default mode network covers large portions of the posterior cingulate and precuneus, angular gyrus, and frontal medial cortex. The corresponding brain map is displayed in the Online resources.

In the default mode network, the effect of stress was moderated by 5 -HTTLPR genotype in the left posterior supramarginal gyrus extending into the angular gyrus (see 
Table 1 Demographic information on the participants, split by 5-HTTLPR genotype

\begin{tabular}{|c|c|c|c|c|c|c|c|}
\hline Variable & S-allele carriers & SD & L-allele homozygotes & $\mathrm{SD}$ & Test-statistic & DF & $P$-value \\
\hline Participants & 284 & & 141 & & & & \\
\hline \multicolumn{8}{|l|}{ Covariates } \\
\hline Amsterdam location & $49.3 \%$ & & $57.4 \%$ & & $X^{2}=2.51$ & 1 & .11 \\
\hline Male sex & $53.5 \%$ & & $62.4 \%$ & & $X^{2}=2.68$ & 1 & .10 \\
\hline Age in years & 17.19 & 3.49 & 17.44 & 3.41 & $\mathrm{~F}=0.47$ & 423 & .49 \\
\hline Parents' years of education & 11.92 & 2.50 & 12.29 & 2.50 & $\mathrm{~F}=1.99$ & 423 & .16 \\
\hline Stress score & 1.55 & 1.18 & 1.75 & 1.17 & $\mathrm{~F}=2.78$ & 423 & .10 \\
\hline Number of stressful live events & 2.00 & 1.52 & 2.21 & 1.56 & $F=1.76$ & 423 & .19 \\
\hline Number of long-term difficulties & 1.03 & 1.41 & 1.29 & 1.50 & $\mathrm{~F}=3.00$ & 410 & .08 \\
\hline Head motion during scanning & 0.67 & 2.18 & 0.79 & 2.75 & $F=0.26$ & 423 & .61 \\
\hline
\end{tabular}

Differences between genotypes in the categorical variables 'location' and 'sex' were analyzed with a Chi-square test; for the other, continuous variables we performed an analysis of variance. $S D$ standard deviation, $D F$ degrees of freedom. Head motion was measured as the root mean squared of the framewise displacement time series

Fig. 1 and Table 2). In this region, S-allele carriers had a more positive correlation between stress and connectivity than Lallele homozygotes (see Fig. 2B).

We did not find any mediation effects, i.e. connectivity patterns in either network did not significantly explain the association between the GxE and ADHD severity.

Given our focus on the GxE, details on the conditional effects of stress and genotype on connectivity, and the association between connectivity and ADHD severity, are presented in the Online resources.

\section{Sensitivity analyses}

Results from the sensitivity analyses can also be found in the Online resources. Briefly, the direction of effects was the same across diagnostic status, testing locations, and age groups.

\section{Discussion}

We investigated whether the interaction between 5-HTTLPR genotype and stress exposure is associated with differences in connectivity of two brain networks involved in the processing of emotional stimuli, the executive control network and default mode network. We combined this with mediation analysis to determine whether connectivity of these networks could explain a previously reported significant association between this GxE and ADHD severity (van der Meer et al. 2014).

In the executive control network, S-allele carriers had a more negative association between the amount of stress exposure and functional connectivity than L-allele homozygotes, whereas they had a more positive relation between stress exposure and connectivity of the posterior hub of the default mode network. While speculative, the reported effects may contribute to the often-reported association of this GxE with neuroticism, rumination, and worrying (Lesch et al. 1996). Higher default mode network connectivity during rest has been tied directly to greater trait rumination (Berman et al. 2011), and lowered cognitive control is also associated with rumination and worrying (Beckwe et al. 2014). The opposing pattern in the two networks is particularly interesting in light of findings suggesting that the anti-correlation between task-positive and task-negative networks is of higher relevance to behavior than network activity per se (Fox et al. 2005). Specifically,

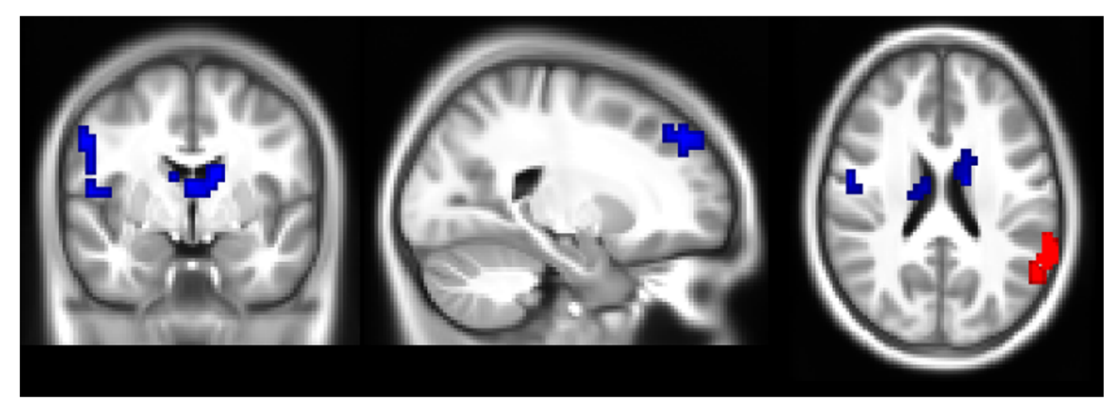

Fig. 1 Location of clusters where the 5-HTTLPR by stress interaction had a significant negative effect on connectivity in the executive control network (blue), and a positive effect on connectivity in the default mode network (red). These maps are overlaid on the sample's average anatomical image, at MNIcoordinates $22,-2,22$ 
Fig. 2 Interaction effect between 5-HTTLPR genotype and stress exposure on connectivity, with S-allele carriers represented by the solid line and points and L-allele homozygotes by the dashed line and open points. a displays the mean of the regression coefficients from the four clusters found in the executive control network, and $\mathbf{b}$ shows the interaction effect found for the cluster in the default mode network a Regression of stress on connectivity of the executive control network

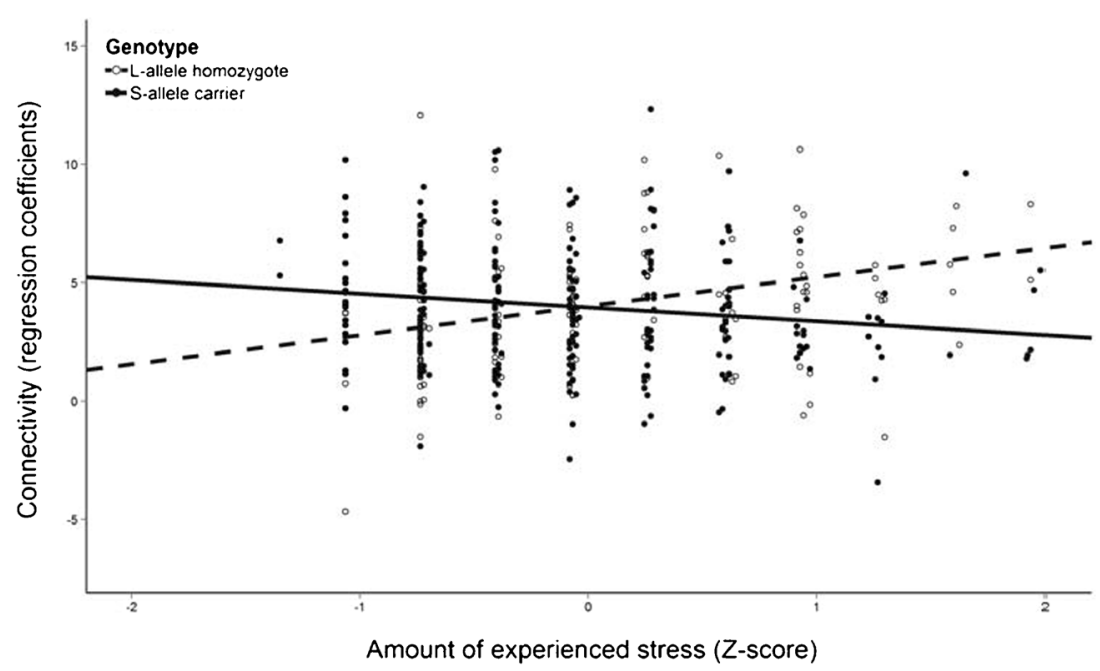

b Regression of stress on connectivity of the default mode network

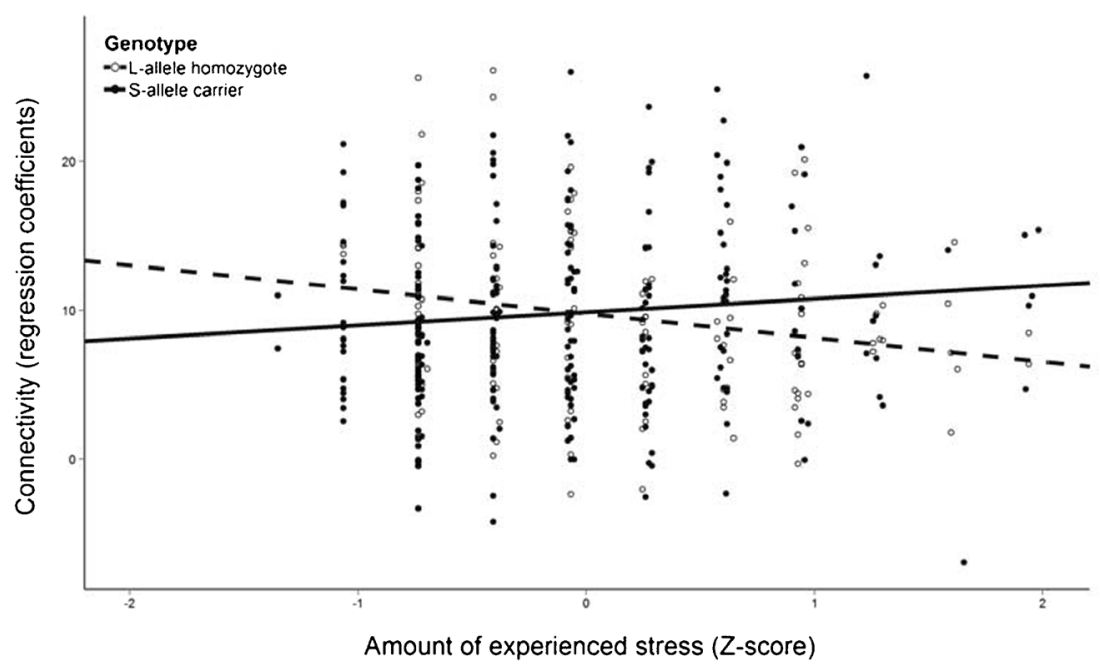

dominance of regions involved in internally-oriented attention over regions involved in externally-oriented attention has been proposed to underlie rumination, impaired attentional control, and cognitive reactivity (Marchetti et al. 2012), and an imbalance between default mode and regulatory networks has been repeatedly found in psychiatric populations characterized by emotional problems (Belleau et al. 2014). Our results may therefore reflect the tendency for S-allele carriers to develop a dysfunctional, internally-focused, cognitive style when exposed to stress (Wells et al. 2010).
Table 2 Information on the clusters where resting-state network connectivity was found to be significantly associated with the gene-environment interaction

\begin{tabular}{llrrrrrr}
\hline RSN & Location & $\mathrm{X}$ & $\mathrm{Y}$ & $\mathrm{Z}$ & Cluster size & Coefficient & ${\text { Cohens } \mathrm{f}^{2}}^{\text {Executive }}$ \\
& Post-, precentral gyrus & -34 & -22 & 36 & 21 & -1.57 & .023 \\
& Frontal pole & 22 & 46 & 40 & 26 & -2.61 & .035 \\
& Post-, precentral gyrus & 54 & -2 & 36 & 47 & -2.33 & .036 \\
& Caudate nucleus, thalamus & -10 & -2 & 16 & 68 & -2.14 & .050 \\
\multirow{2}{*}{ DMN } & Supramarginal gyrus & -62 & -50 & 24 & 22 & 2.62 & .033 \\
\hline
\end{tabular}

$\mathrm{X}, \mathrm{Y}, \mathrm{Z}$ coordinates are in MNI-space in $\mathrm{mm}$, and represent the peak of the cluster. The anatomical labels are according to the Harvard-Oxford atlas. RSN Resting-state network, DMN Default-mode network, MNI Montreal Neurological Institute. Cluster size indicates number of voxels in that cluster, at $4 \mathrm{~mm}$ isotropic resolution 
We found lowered functional connectivity of the precentral gyrus extending into the postcentral gyrus, frontal pole, and caudate nucleus with the executive control network, all regions previously associated with this GxE (Canli et al. 2005, 2006; van der Meer et al. 2015) and all important for cognitive control when faced with emotional stimuli. The precentral gyrus is active during cognitive reappraisal of distressing stimuli (Belden et al. 2014). The postcentral gyrus has shown higher activity specifically during the presentation of negative words in an emotional Stroop task, in a study that showed that tryptophan depletion (i.e., lowered serotonin signaling) improved task performance (Evers et al. 2006). The frontal pole is central to inhibiting automatic emotional responses (Volman et al. 2011). And lastly, connectivity of the caudate nucleus with the prefrontal cortex has been found to explain successful emotion regulation through reappraisal (Wager et al. 2008). We further found a significantly more positive relation between stress exposure and connectivity of the supramarginal gyrus within the default mode network for S-allele carriers compared to L-allele homozygotes, in line with previous reports of effects of this GxE on the superior parietal lobe (Canli and Lesch 2007). Heightened connectivity of this region may be tied to its role in self-referential processing (Silani et al. 2013), given the association of 5-HTTLPR and stress exposure with rumination and worrying (Clasen et al. 2011; Schinka et al. 2004), traits common in individuals with ADHD (Nigg et al. 2002; Shaw et al. 2014). The lateral parietal lobe is also linked to post-traumatic stress disorder (Morey et al. 2011; Liberzon and Sripada 2007) and known to facilitate episodic memory retrieval (Davidson et al. 2008), supporting the notion that this finding relates to rumination.

The reported connectivity patterns did not significantly explain why S-allele carriers have a stronger association between stress exposure and ADHD severity than L-allele homozygotes. Whereas we previously reported that gray matter volume in the frontal pole mediated the effects of this GxE on ADHD (van der Meer et al. 2015), functional connectivity of this region within the executive control network did not. Lack of significant mediation findings in this study indicates that the effect of this GxE on connectivity patterns within either network is insufficient to explain its relation with ADHD severity. It may be the case though, that a combination of connectivity patterns within several networks is necessary to significantly contribute to behavior as complex as the ADHD phenotype. For instance, interference of task-irrelevant thoughts through decreased suppression of default mode network activity has been suggested to contribute to lower cognitive performance of individuals with ADHD (Sonuga-Barke and Castellanos 2007), which may result both from lowered connectivity of the executive control network and stronger connectivity of the default mode network. This combined effect may be investigated in future studies analyzing cross-network connectivity. It may also be captured during task performance taxing both cognitive control and emotion processing. The emotional Stroop task seems particularly suitable for this, as the brain regions found in the current study have all been reported to be active during this task (Veroude et al. 2013; Evers et al. 2006; Sadeh et al. 2011; Malhi et al. 2005). In addition, 5HTTLPR genotype has been shown to influence recruitment of cognitive control brain regions during incongruent trials (Stollstorff et al. 2013), and individuals with ADHD have shown higher interference by negatively-valenced words compared to healthy controls (Posner et al. 2011).

Strengths of this study include a large sample size, use of multiple informants to determine ADHD phenotype, and the hypothesis-driven study of robust and reliable brain networks using multivariate statistics. A limitation is the observational, cross-sectional design of our study, preventing strong inferences about causality. For instance, it could be the case that the reported neural differences produce maladaptive behavior, which in turn may lead to the experience of more stressful live events. While animal studies have provided causal evidence for the brain of S-allele carriers being more strongly affected by exposure to stress (Spinelli et al. 2007), longitudinal studies or studies making use of 'natural experiments' (Rutter 2007), are needed to confirm this causality in humans.

In conclusion, the interaction between 5-HTTLPR and stress exposure is associated with decreased connectivity in the executive control network and increased connectivity within the default mode network. Although in need of replication, the reported effects may contribute to the association of this $\mathrm{GxE}$ with a range of pathological behaviors due to lowered cognitive control and enhanced emotional reactivity. Ultimately, this type of research may contribute to the prevention and treatment of stress-related pathological behavior, by identifying those most at risk and by providing knowledge on the neural mechanisms involved.

Acknowledgments We acknowledge the department of Pediatrics of the VU University Medical Center for having the opportunity to use the mock scanner for preparation of our participants.

\section{Compliance with ethical standards}

Funding This work was supported by National Institutes of Health (NIH) Grant R01MH62873 (to Stephen V. Faraone), Nederlandse Organisatie voor Wetenschappelijk Onderzoek (NWO) Large Investment Grant 1,750,102,007,010 and NWO Brain \& Cognition an Integrative Approach grant (433-09-242) (to Jan. Buitelaar), and grants from Radboud University Nijmegen Medical Center, University Medical Center Groningen and Accare, and VU University Amsterdam. The research leading to these results also received funding from the European Community's Seventh Framework Programme (FP7/2007-2013) under grant agreement numbers 278,948 (TACTICS), 602,450 (IMAGEMEND) and $n^{\circ} 602,805$ (Aggressotype), and from the European Community's Horizon 2020 Programme (H2020/2014-2020) under grant agreement $n^{\circ} 643,051$ (MiND). Barbara Franke is supported by a Vici grant from NWO (grant number 016-130-669). In addition, Jan. Buitelaar and Barbara Franke are supported by a grant for the ENIGMA Consortium (grant number U54 EB020403) from the BD2K Initiative of a cross-NIH partnership. 
Conflict of interest Barbara Franke has received a speaker fee from Merz. Pieter J. Hoekstra has received an unrestricted research grant from Shire and has been member of the advisory boards of Shire and Eli Lilly. Jaap Oosterlaan has received an unrestricted investigator initiated research grant from Shire pharmaceuticals. Jan. K. Buitelaar has been in the past 3 years a consultant to / member of advisory board of / and/or speaker for Janssen Cilag BV, Eli Lilly, Shire, Novartis, Roche and Servier. He is not an employee of any of these companies, and not a stock shareholder of any of these companies. In the past year, Stephen V. Faraone received income, travel expenses and/or research support from Pfizer, Ironshore, Shire, Akili Interactive Labs, Alcobra, VAYA Pharma, and SynapDx and research support from the National Institutes of Health $(\mathrm{NIH})$. His institution is seeking a patent for the use of sodium-hydrogen exchange inhibitors in the treatment of ADHD. In previous years, he received consulting fees or was on Advisory Boards or participated in continuing medical education programs sponsored by: Shire, Alcobra, Otsuka, McNeil, Janssen, Novartis, Pfizer and Eli Lilly. Dr. Faraone receives royalties from books published by Guilford Press: Straight Talk about Your Child's Mental Health and Oxford University Press: Schizophrenia: The Facts. All other authors report no competing interests.

Ethical approval All procedures performed in studies involving human participants were in accordance with the ethical standards of the institutional and/or national research committee and with the 1964 Helsinki declaration and its later amendments or comparable ethical standards.

This article does not contain any studies with animals performed by any of the authors.

Open Access This article is distributed under the terms of the Creative Commons Attribution 4.0 International License (http:// creativecommons.org/licenses/by/4.0/), which permits unrestricted use, distribution, and reproduction in any medium, provided you give appropriate credit to the original author(s) and the source, provide a link to the Creative Commons license, and indicate if changes were made.

\section{References}

Barnhofer, T., \& Chittka, T. (2010). Cognitive reactivity mediates the relationship between neuroticism and depression. Behaviour Research and Therapy, 48(4), 275-281.

Beckmann, C. F., Mackay, C. E., Filippini, N., \& Smith, S. M. (2009). Group comparison of resting-state FMRI data using multi-subject ICA and dual regression. NeuroImage, 47(Suppl 1), S148.

Beckwe, M., Deroost, N., Koster, E. H., De Lissnyder, E., \& De Raedt, R. (2014). Worrying and rumination are both associated with reduced cognitive control. Psychological Research, 78(5), 651-660. doi:10.1007/s00426-013-0517-5.

Belden, A. C., Luby, J. L., Pagliaccio, D., \& Barch, D. M. (2014). Neural activation associated with the cognitive emotion regulation of sadness in healthy children. Developmental Cognitive Neuroscience, 9, 136-147.

Belleau, E. L., Taubitz, L. E., \& Larson, C. L. (2014). Imbalance of default mode and regulatory networks during externally focused processing in depression. Social cognitive and affective neuroscience. doi: $10.1093 / \mathrm{scan} / \mathrm{nsu} 117$.

Berman, M. G., Peltier, S., Nee, D. E., Kross, E., Deldin, P. J., \& Jonides, J. (2011). Depression, rumination and the default network. Social Cognitive and Affective Neuroscience, 6(5), 548-555. doi: $10.1093 /$ scan $/$ nsq080
Borg, J., Henningsson, S., Saijo, T., Inoue, M., Bah, J., Westberg, L., et al. (2009). Serotonin transporter genotype is associated with cognitive performance but not regional 5-HT1A receptor binding in humans. The International Journal of Neuropsychopharmacology / Official Scientific Journal of the Collegium Internationale Neuropsychopharmacologicum (CINP), 12(6), 783-792. doi:10.1017/S1461145708009759.

Bosch, N. M., Riese, H., Reijneveld, S. A., Bakker, M. P., Verhulst, F. C., Ormel, J., et al. (2012). Timing matters: long term effects of adversities from prenatal period up to adolescence on adolescents' cortisol stress response. The TRAILS study. Psychoneuroendocrinology, 37(9), 1439-1447. doi:10.1016/j.psyneuen.2012.01.013.

Brookes, K., Xu, X., Chen, W., Zhou, K., Neale, B., Lowe, N., et al. (2006). The analysis of 51 genes in DSM-IV combined type attention deficit hyperactivity disorder: association signals in DRD4, DAT1 and 16 other genes. Molecular Psychiatry, 11(10), 934-953. doi:10.1038/sj.mp.4001869.

Broyd, S. J., Demanuele, C., Debener, S., Helps, S. K., James, C. J., \& Sonuga-Barke, E. J. (2009). Default-mode brain dysfunction in mental disorders: a systematic review. Neuroscience and Biobehavioral Reviews, 33(3), 279-296. doi:10.1016/j. neubiorev.2008.09.002.

Buckner, R. L., Andrews-Hanna, J. R., \& Schacter, D. L. (2008). The brain's default network: anatomy, function, and relevance to disease. Annals of the New York Academy of Sciences, 1124, 1-38. doi:10.1196/annals.1440.011.

Buis, M. L. (2010). Scaling levels of education. Faculty of Social Sciences: VU-University Amsterdam.

Bush, G., Frazier, J. A., Rauch, S. L., Seidman, L. J., Whalen, P. J., Jenike, M. A., et al. (1999). Anterior cingulate cortex dysfunction in attention-deficit/hyperactivity disorder revealed by fMRI and the counting Stroop. Biological Psychiatry, 45(12), 1542-1552.

Canli, T., \& Lesch, K. P. (2007). Long story short: the serotonin transporter in emotion regulation and social cognition. Nature Neuroscience, 10(9), 1103-1109. doi:10.1038/nn1964.

Canli, T., Omura, K., Haas, B. W., Fallgatter, A., Constable, R. T., \& Lesch, K. P. (2005). Beyond affect: a role for genetic variation of the serotonin transporter in neural activation during a cognitive attention task. Proceedings of the National Academy of Sciences of the United States of America, 102(34), 12224-12229. doi:10.1073 /pnas.0503880102.

Canli, T., Qiu, M., Omura, K., Congdon, E., Haas, B. W., Amin, Z., et al. (2006). Neural correlates of epigenesis. Proceedings of the National Academy of Sciences of the United States of America, 103(43), 16033-16038. doi:10.1073/pnas.0601674103.

Caspi, A., \& Moffitt, T. E. (2006). Gene-environment interactions in psychiatry: joining forces with neuroscience. Nature Reviews Neuroscience, 7(7), 583-590. doi:10.1038/nrn1925.

Caspi, A., Hariri, A. R., Holmes, A., Uher, R., \& Moffitt, T. E. (2010). Genetic sensitivity to the environment: the case of the serotonin transporter gene and its implications for studying complex diseases and traits. The American Journal of Psychiatry, 167(5), 509-527. doi:10.1176/appi.ajp.2010.09101452.

Castellanos, F. X., Margulies, D. S., Kelly, C., Uddin, L. Q., Ghaffari, M., Kirsch, A., et al. (2008). Cingulate-precuneus interactions: a new locus of dysfunction in adult attentiondeficit/hyperactivity disorder. Biological Psychiatry, 63(3), 332-337. doi:10.1016/j.biopsych.2007.06.025.

Clasen, P. C., Wells, T. T., Knopik, V. S., McGeary, J. E., \& Beevers, C. G. (2011). 5-HTTLPR and BDNF Val66Met polymorphisms moderate effects of stress on rumination. Genes, Brain and Behavior, 10(7), 740-746. doi:10.1111/j.1601-183X.2011.00715.x.

Conners, C. K., Sitarenios, G., Parker, J. D., \& Epstein, J. N. (1998). The revised Conners' parent rating scale (CPRS-R): factor structure, reliability, and criterion validity. Journal of Abnormal Child Psychology, 26(4), 257-268. 
Damoiseaux, J. S., Rombouts, S. A. R. B., Barkhof, F., Scheltens, P., Stam, C. J., Smith, S. M., et al. (2006). Consistent restingstate networks across healthy subjects. Proceedings of the National Academy of Sciences, 103(37), 13848-13853. doi:10.1073/pnas.0601417103.

Davidson, P. S. R., Anaki, D., Ciaramelli, E., Cohn, M., Kim, A. S. N., Murphy, K. J., et al. (2008). Does lateral parietal cortex support episodic memory?: evidence from focal lesion patients. Neuropsychologia, 46(7), 1743-1755. doi:10.1016/j.neuropsychologia.2008.01.011.

Evers, E., Van der Veen, F., Jolles, J., Deutz, N., \& Schmitt, J. (2006). Acute tryptophan depletion improves performance and modulates the BOLD response during a Stroop task in healthy females. NeuroImage, 32(1), 248-255.

Filippini, N., MacIntosh, B. J., Hough, M. G., Goodwin, G. M., Frisoni, G. B., Smith, S. M., et al. (2009). Distinct patterns of brain activity in young carriers of the APOE- 44 allele. Proceedings of the National Academy of Sciences, 106(17), 7209-7214.

Fischer, A. G., Endrass, T., Reuter, M., Kubisch, C., \& Ullsperger, M. (2015). Serotonin reuptake inhibitors and serotonin transporter genotype modulate performance monitoring functions but not their electrophysiological correlates. The Journal of Neuroscience : the Official Journal of the Society for Neuroscience, 35(21), 81818190. doi:10.1523/JNEUROSCI.5124-14.2015.

Fox, M. D., Snyder, A. Z., Vincent, J. L., Corbetta, M., Van Essen, D. C., $\&$ Raichle, M. E. (2005). The human brain is intrinsically organized into dynamic, anticorrelated functional networks. Proceedings of the National Academy of Sciences of the United States of America, 102(27), 9673-9678.

Gizer, I. R., Ficks, C., \& Waldman, I. D. (2009). Candidate gene studies of ADHD: a meta-analytic review. Human Genetics, 126(1), 51-90. doi:10.1007/s00439-009-0694-x.

Hayes, A. F. (2013). Introduction to mediation, moderation, and conditional process analysis: a regression-based approach (Vol. Book, Whole): Guilford Press.

Homberg, J. R., \& Lesch, K. P. (2011). Looking on the bright side of serotonin transporter gene variation. Biological Psychiatry, 69(6), 513-519. doi:10.1016/j.biopsych.2010.09.024.

Hu, X. Z., Lipsky, R. H., Zhu, G., Akhtar, L. A., Taubman, J., Greenberg, B. D., et al. (2006). Serotonin transporter promoter gain-of-function genotypes are linked to obsessive-compulsive disorder. American Journal of Human Genetics, 78(5), 815-826. doi:10.1086/503850.

Jenkinson, M., Bannister, P., Brady, M., \& Smith, S. (2002). Improved optimization for the robust and accurate linear registration and motion correction of brain images. NeuroImage, 17(2), 825-841.

Kaufman, J., Birmaher, B., Brent, D., Rao, U., Flynn, C., Moreci, P., et al. (1997). Schedule for affective disorders and schizophrenia for school-age children-present and lifetime version (K-SADSPL): initial reliability and validity data. Journal of the American Academy of Child and Adolescent Psychiatry, 36(7), 980-988. doi:10.1097/00004583-199707000-00021.

Kunisato, Y., Okamoto, Y., Okada, G., Aoyama, S., Demoto, Y., Munakata, A., et al. (2011). Modulation of default-mode network activity by acute tryptophan depletion is associated with mood change: a resting state functional magnetic resonance imaging study. Neuroscience Research, 69(2), 129-134.

Laird, A. R., Fox, P. M., Eickhoff, S. B., Turner, J. A., Ray, K. L., McKay, D. R., et al. (2011). Behavioral interpretations of intrinsic connectivity networks. Journal of Cognitive Neuroscience, 23(12), 40224037. doi:10.1162/jocn_a_00077.

Lesch, K. P., Bengel, D., Heils, A., Sabol, S. Z., Greenberg, B. D., Petri, S., et al. (1996). Association of anxiety-related traits with a polymorphism in the serotonin transporter gene regulatory region. Science (New York, N.Y.), 274(5292), 1527-1531.

Levy, F., Hay, D. A., McStephen, M., Wood, C., \& Waldman, I. (1997). Attention-deficit hyperactivity disorder: a category or a continuum? Genetic analysis of a large-scale twin study. Journal of the American
Academy of Child and Adolescent Psychiatry, 36(6), 737-744. doi:10.1097/00004583-199706000-00009.

Liberzon, I., \& Sripada, C. S. (2007). The functional neuroanatomy of PTSD: a critical review. Progress in Brain Research, 167, 151-169.

Luman, M., Oosterlaan, J., \& Sergeant, J. A. (2005). The impact of reinforcement contingencies on $\mathrm{AD} / \mathrm{HD}$ : a review and theoretical appraisal. Clinical Psychology Review, 25(2), 183-213. doi:10.1016/j.cpr.2004.11.001.

Malhi, G. S., Lagopoulos, J., Sachdev, P. S., Ivanovski, B., \& Shnier, R. (2005). An emotional Stroop functional MRI study of euthymic bipolar disorder. Bipolar Disorders, 7(s5), 58-69.

Marchetti, I., Koster, E. H., Sonuga-Barke, E. J., \& De Raedt, R. (2012). The default mode network and recurrent depression: a neurobiological model of cognitive risk factors. Neuropsychology Review, 22(3), 229-251. doi:10.1007/s11065-012-9199-9.

Martel, M., Nigg, J., \& von Eye, A. (2009). How do trait dimensions map onto ADHD symptom domains? Journal of Abnormal Child Psychology, 37(3), 337-348. doi:10.1007/s10802-008-9255-3.

Meyer-Lindenberg, A. (2009). Neural connectivity as an intermediate phenotype: brain networks under genetic control. Human Brain Mapping, 30(7), 1938-1946. doi:10.1002/hbm.20639.

Mitchell, J., Benson, J., Knouse, L., Kimbrel, N., \& Anastopoulos, A. (2013). Are negative automatic thoughts associated with ADHD in adulthood? Cognitive Therapy and Research, 37(4), 851-859. doi:10.1007/s10608-013-9525-4.

Morey, R. A., Hariri, A. R., Gold, A. L., Hauser, M. A., Munger, H. J., Dolcos, F., et al. (2011). Serotonin transporter gene polymorphisms and brain function during emotional distraction from cognitive processing in posttraumatic stress disorder. [journal article]. BMC Psychiatry, 11(1), 1-13. doi:10.1186/1471-244x-11-76.

Munafo, M. R., Brown, S. M., \& Hariri, A. R. (2008). Serotonin transporter (5-HTTLPR) genotype and amygdala activation: a meta-analysis. Biological Psychiatry, 63(9), 852-857.

Nigg, J. T., John, O. P., Blaskey, L. G., Huang-Pollock, C. L., Willicut, E. G., Hinshaw, S. P., et al. (2002). Big five dimensions and ADHD symptoms: links between personality traits and clinical symptoms. Journal of Personality and Social Psychology, 83(2), 451.

Oldehinkel, A. J., Verhulst, F. C., \& Ormel, J. (2008). Low heart rate: a marker of stress resilience. The TRAILS study. Biological Psychiatry, 63(12), 1141-1146. doi:10.1016/j.biopsych.2007.12.006.

Oldehinkel, M., Francx, W., Beckmann, C. F., Buitelaar, J. K., \& Mennes, M. (2013). Resting state FMRI research in child psychiatric disorders. European Child \& Adolescent Psychiatry, 22(12), 757-770. doi:10.1007/s00787-013-0480-0.

Owens, M., Goodyer, I. M., Wilkinson, P., Bhardwaj, A., Abbott, R., Croudace, T., et al. (2012). 5-HTTLPR and early childhood adversities moderate cognitive and emotional processing in adolescence. PloS One, 7(11), e48482. doi:10.1371/journal.pone.0048482.

Parker, J. D., Majeski, S. A., \& Collin, V. T. (2004). ADHD symptoms and personality: relationships with the five-factor model. Personality and Individual Differences, 36(4), 977-987.

Pergamin-Hight, L., Bakermans-Kranenburg, M. J., van Ijzendoorn, M. H., \& Bar-Haim, Y. (2012). Variations in the promoter region of the serotonin transporter gene and biased attention for emotional information: a meta-analysis. Biological Psychiatry, 71(4), 373-379. doi:10.1016/j.biopsych.2011.10.030.

Pezawas, L., Meyer-Lindenberg, A., Drabant, E. M., Verchinski, B. A., Munoz, K. E., Kolachana, B. S., et al. (2005). 5-HTTLPR polymorphism impacts human cingulate-amygdala interactions: a genetic susceptibility mechanism for depression. Nature Neuroscience, 8(6), 828-834. doi:10.1038/nn1463.

Posner, J., Maia, T. V., Fair, D., Peterson, B. S., Sonuga-Barke, E. J., \& Nagel, B. J. (2011). The attenuation of dysfunctional emotional processing with stimulant medication: an fMRI study of adolescents with ADHD. Psychiatry Research: Neuroimaging, 193(3), 151-160. 
Pruim, R. H., Mennes, M., van Rooij, D., Llera, A., Buitelaar, J. K., \& Beckmann, C. F. (2015). ICA-AROMA: A robust ICA-based strategy for removing motion artifacts from fMRI data. NeuroImage

Puig, M. V., \& Gulledge, A. T. (2011). Serotonin and prefrontal cortex function: neurons, networks, and circuits. Molecular Neurobiology, 44(3), 449-464.

R Core Team (2012). $R$ : A language and environment for statistical computing. Vienna, Austria: R Foundation for Statistical Computing.

Raichle, M. E., MacLeod, A. M., Snyder, A. Z., Powers, W. J., Gusnard, D. A., \& Shulman, G. L. (2001). A default mode of brain function. Proceedings of the National Academy of Sciences of the United States of America, 98(2), 676-682. doi:10.1073/pnas.98.2.676.

Roiser, J. P., Müller, U., Clark, L., \& Sahakian, B. J. (2007). The effects of acute tryptophan depletion and serotonin transporter polymorphism on emotional processing in memory and attention. The International Journal of Neuropsychopharmacology, 10(04), 449-461.

Rutter, M. (2007). Proceeding from observed correlation to causal inference: the use of natural experiments. Perspectives on Psychological Science, 2(4), 377-395. doi:10.1111/j.1745-6916.2007.00050.x.

Sadeh, N., Spielberg, J. M., Heller, W., Herrington, J. D., Engels, A. S., Warren, S. L., et al. (2011). Emotion disrupts neural activity during selective attention in psychopathy. Social Cognitive and Affective Neuroscience. doi:10.1093/scan/nsr092.

Schinka, J., Busch, R., \& Robichaux-Keene, N. (2004). A meta-analysis of the association between the serotonin transporter gene polymorphism (5-HTTLPR) and trait anxiety. Molecular Psychiatry, 9(2), 197-202.

Selvaraj, S., Godlewska, B. R., Norbury, R., Bose, S., Turkheimer, F., Stokes, P., et al. (2011). Decreased regional gray matter volume in $\mathrm{S}^{\prime}$ allele carriers of the 5-HTTLPR triallelic polymorphism. Molecular Psychiatry, 16(5), 471, 472-471, 473. doi:10.1038/mp.2010.112.

Selya, A. S., Rose, J. S., Dierker, L. C., Hedeker, D., \& Mermelstein, R. J. (2012). A practical guide to calculating Cohen's $\mathrm{f}(2)$, a measure of local effect size, from PROC MIXED. Frontiers in Psychology, 3, 111. doi:10.3389/fpsyg.2012.00111.

Shaw, P., Stringaris, A., Nigg, J., \& Leibenluft, E. (2014). Emotion dysregulation in attention deficit hyperactivity disorder. The American Journal of Psychiatry, 171(3), 276-293. doi:10.1176/appi. ajp.2013.13070966.

Silani, G., Lamm, C., Ruff, C. C., \& Singer, T. (2013). Right supramarginal gyrus is crucial to overcome emotional egocentricity bias in social judgments. The Journal of Neuroscience, 33(39), 15466-15476.

Smith, S. M., Fox, P. T., Miller, K. L., Glahn, D. C., Fox, P. M., Mackay, C. E., et al. (2009). Correspondence of the brain's functional architecture during activation and rest. Proceedings of the National Academy of Sciences of the United States of America, 106(31), 13040-13045. doi:10.1073/pnas.0905267106.

Sodhi, M. S., \& Sanders-Bush, E. (2004). Serotonin and brain development. International Review of Neurobiology, 59, 111-174. doi:10.1016/S0074-7742(04)59006-2.
Sonuga-Barke, E. J., \& Castellanos, F. X. (2007). Spontaneous attentional fluctuations in impaired states and pathological conditions: a neurobiological hypothesis. Neuroscience and Biobehavioral Reviews, 31(7), 977-986.

Spinelli, S., Schwandt, M. L., Lindell, S. G., Newman, T. K., Heilig, M., Suomi, S. J., et al. (2007). Association between the recombinant human serotonin transporter linked promoter region polymorphism and behavior in rhesus macaques during a separation paradigm. Development and Psychopathology, 19(4), 977-987. doi:10.1017 /S095457940700048X.

Stollstorff, M., Munakata, Y., Jensen, A. P., Guild, R. M., Smolker, H. R., Devaney, J. M., et al. (2013). Individual differences in emotioncognition interactions: emotional valence interacts with serotonin transporter genotype to influence brain systems involved in emotional reactivity and cognitive control. Frontiers in Human Neuroscience, 7.

van der Meer, D., Hartman, C. A., Richards, J., Bralten, J. B., Franke, B., Oosterlaan, J., et al. (2014). The serotonin transporter gene polymorphism 5-HTTLPR moderates the effects of stress on attention-deficit/hyperactivity disorder. Journal of Child Psychology and Psychiatry, and Allied Disciplines. doi:10.1111/jcpp.12240.

van der Meer, D., Hoekstra, P. J., Zwiers, M., Mennes, M., Schweren, L. J., Franke, B., et al. (2015). Brain correlates of the interaction between 5-HTTLPR and psychosocial stress mediating attention deficit hyperactivity disorder severity. The American Journal of Psychiatry, 172(8), 768-775. doi:10.1176/appi.ajp.2015.14081035.

Veroude, K., Jolles, J., Croiset, G., \& Krabbendam, L. (2013). Changes in neural mechanisms of cognitive control during the transition from late adolescence to young adulthood. Developmental Cognitive Neuroscience, 5, 63-70.

Volman, I., Roelofs, K., Koch, S., Verhagen, L., \& Toni, I. (2011). Anterior prefrontal cortex inhibition impairs control over social emotional actions. Current biology, 21(20), 1766-1770. doi:10.1016/j.cub.2011.08.050.

von Rhein, D., Mennes, M., van Ewijk, H., Groenman, A. P., Zwiers, M. P., Oosterlaan, J., et al. (2014). The NeuroIMAGE study: a prospective phenotypic, cognitive, genetic and MRI study in children with attention-deficit/hyperactivity disorder. Design and descriptives. European Child \& Adolescent Psychiatry. doi:10.1007/s00787-014-0573-4.

Wager, T. D., Davidson, M. L., Hughes, B. L., Lindquist, M. A., \& Ochsner, K. N. (2008). Prefrontal-subcortical pathways mediating successful emotion regulation. Neuron, 59(6), 1037-1050. doi:10.1016/j.neuron.2008.09.006.

Wells, T. T., Beevers, C. G., \& McGeary, J. E. (2010). Serotonin transporter and BDNF genetic variants interact to predict cognitive reactivity in healthy adults. Journal of Affective Disorders, 126(1), 223-229.

Whitfield-Gabrieli, S., \& Ford, J. M. (2012). Default mode network activity and connectivity in psychopathology. Annual Review of Clinical Psychology, 8, 49-76. 